\title{
Statistical properties of staircase estimates ${ }^{1}$
}

\author{
RICHARD M. ROSE, ${ }^{2}$ DAVIDA Y. TELLER, AND PAULA RENDLEMAN ${ }^{3}$ \\ UNIVERSITY OF WASHINGTON
}

The bias and variability of staircase estimators were studied by means of repeated computer simulations of staircase runs. Both forced-choice and yes-no staircases were simulated. The influence of the shape of the psychometric function, the location and spacing of the stimuli, the number of trials in a run, and the method of deriving the estimate from the data are discussed. The forced-choice staircase is compared to the yes-no staircase, and the limitations of the simulation procedure are outlined.

A sizable proportion of psychophysical procedures are designed to determine the level of an independent variable that leads to some predetermined response probability. ${ }^{4}$ For example, an E may wish to determine the stimulus intensity necessary for the $S$ to say, "Yes, I detect the stimulus," on $50 \%$ of the trials, or the intensity necessary for him to report the location of the stimulus with some specified probability between $50 \%$ and $100 \%$. The intensity level found in such an experiment is often termed the "threshold." Campbell and Lasky (1968) report that three out of four papers in the Journal of the Acoustical Society of America in 1966 and 1967 dealt with measurements of this sort.

The methods that psychologists have devised to measure thresholds can be divided into two classes constant stimulus or fixed psychophysical methods, and adaptive or titration methods. In the former, repeated presentations are made at two or more stimulus intensities, and the probability of detecting the stimulus is estimated for each intensity level. Then the best fitting psychometric function is found, and the desired intensity level read off. Adaptive methods, on the other hand, do not involve separate tests at more than one point along the psychometric function. Instead, one or a small number of trials are run at a given level, and the responses made on those trials determine the next stimulus level. The decision rules for selection of the intensities are designed so that after a series of steps the intensity level will, more often than not, tend toward the threshold.

A variety of adaptive methods for estimating thresholds exist, e.g., the block up-and-down methods (Stuckey, Hutton, \& Campbell, 1966), several procedures, sometimes referred to as PEST (parameter estimation by sequential testing) procedures (Taylor \& Creelman, 1967; Pollack, 1968), and staircase methods, which are considered in this paper. The latter type of procedure was called the up-and-down rule by Dixon and Mood (1948) and the staircase procedure by Cornsweet (1962). It has been widely used in the study of vision (e.g., Blough, 1955 Cornsweet \& Pinsker, 1965; Heinemann, 1961; Teller, 1965; Thomas, Padilla, \& Rourke, 1969) and in some auditory threshold studies (e.g., Brown, 1965; Levitt, 1964).

In its simplest version the staircase method is used to estimate the $50 \%$ point on a psychometric function in a threshold experiment that uses the yes-no procedure. The $E$ selects a sequence of stimulus levels, equally spaced along some stimulus scale, such as the intensity of a briefly presented spot of light. On each trial, the $E$ presents a stimulus. If the response is correct (incorrect) he presents the next dimmer (brighter) spot in the sequence. Typically some predetermined number of trials are run, the first few trials of the run are discarded, and the threshold estimate is found by averaging the levels of the stimuli presented on the remaining trials. On the assumption that a stable psychometric function exists, it can be shown that this procedure provides an asymptotically unbiased estimator of the $50 \%$ point of that function (Wetherill, 1963).

The yes-no procedure, like other procedures in which the stimulus is presented on every trial, has one important disadvantage: the $S$ is free to set his own criterion for making the "yes" response (Green \& Swets, 1966). Semb and Saslow (1966) have shown that this problem can persist when the yes-no procedure is used in conjunction with a staircase method. For this reason, many Es now use symmetric forced-choice procedures, in which the criterion can be brought under the control of the $\mathbf{E}$. In a k-alternative forced-choice procedure, the stimulus to be detected is presented in exactly one of $k$ intervals, and the $S$ chooses the interval in which he judges the stimulus is most likely to have occurred. With this procedure, it is possible to use feedback to inform him of the correctness of his response, a procedure that is observed to produce greater stability in the S's criterion than is obtained with yes-no procedures (Green \& Swets, 1966). The forced-choice staircase procedure is most frequently used with just two alternatives. For these reasons, we have chosen to study the properties of the staircase method used in conjunction with either the yes-no or the two-alternative forced-choice procedure.

One could, in principle, evaluate the statistical properties of estimates gleaned from staircase data by the lengthy method of running a single $S$ through a large number of staircase runs under constant conditions. We have chosen, instead, to study these properties by simulating repeated staircase runs on a computer. In particular, we study the bias and variability in staircase estimates as a function of the number of trials in a run, the spacing of the stimuli, the method of calculating the estimate, and the shape of the psychometric function.

In the following -sections, we first describe the assumptions that our program makes about the behavior of our statistical $S$ ("stat-S"), and then present a detailed description of the varieties of the staircase method we have studied. Finally, a summary of the results of the simulations is presented and their implications discussed.

\section{PSYCHOPHYSICAL ASSUMPTIONS : THE STAT-SUBJECT}

In the most thorough fixed psychophysical methods, with the yes-no procedure, a sizable block of trials is run at each of several stimulus levels, and the proportion of "yes" responses is plotted as a function of stimulus level. The resulting psychometric function is typically found to resemble the cumulative normal distribution function. Other psychometric functions have been suggested by theoreticians. For example, the neural quantum model of Stevens, Morgan, and Volkmann (1941) generates a linear psychometric function-i.e., a function that takes on the value 0 for all stimulus levels less than some level, $\mathrm{I}_{0}$, takes on the value 1 for all stimulus levels greater than a higher level, $I_{1}$, and rises linearly from 0 to 1 in the interval $\left(I_{0}, I_{1}\right)$. This paper compares staircase data generated by both cumulative normal and linear psychome tric functions.

When the forced-choice procedure is used, the psychometric function is that 
function relating the proportion of correct responses to stimulus level. Clearly, even for exceedingly weak stimuli, the probability of a correct response is at least 0.5 because the $S$ can be correct that of ten by guessing. For stronger stimuli, the function lies in the interval, $(0.5,1)$. When simulations of forced-choice staircase procedures are presented, they have been generated under the assumption of either a cumulative normal or a linear psychometric function as described for the yes-no procedure, except that the lower asymptote of the function is taken to be 0.5 .

The psychometric function is used by the computer program in the following way: Whenever a stimulus, $i$, is presented, the computer finds the associated probability, $p(i)$, on the psychometric function and then generates a correct response (or a "yes" response, as appropriate) with probability $p(i)$, by consulting a random number generator. Further, we assume that successive responses are statistically independent; i.e., we assume no sequential response biases. Implicit in our approach is the assumption that there is no effect of learning, fatigue, or criterion shift that would alter the shape or location of the psychometric function.

\section{VARIETIES OF}

\section{STAIRCASE METHODS STUDIED}

There exist practically as many variants of the staircase procedure as there are experiments that have used it. Precise specification of a threshold estimator requires a complete set of instructions for collecting and processing the staircase data.

\section{Rules for Changing the Stimulus Level}

When a staircase is run, the stimulus intensity on any given trial, $n$, depends on the S's responses on one or more preceding trials. The procedure for changing the stimulus levels from trial to trial may be called the "rule" for the staircase.

With the yes-no procedure, the rule typically used is simple: If the $S$ responds "yes" on Trial $n-1$, the next lower value of the stimulus is presented on Trial $n$; if the $S$ responds "no" on Trial $n-1$, the next higher intensity level is presented on Trial n (Cornsweet, 1962). We have adopted this rule for all our simulations using the yes-no procedure. This procedure tends, on the average, to move the stimulus selected toward that level nearest to the $50 \%$ point on the psychometric function.

Wetherill and Levitt (1965) present seven rules, each of which can be used to estimate a different point on the psychometric function. For the two-alternative forced-choice case, one typically wishes to estimate the $75 \%$ point on the psychometric function. We have selected Wetherill and Levitt's Rule 1, which estimates the 0.707 point on the psychometric function. This rule has been selected for two reasons. First, it is one frequently used in practice (see, e.g., Heinemann, 1961; Teller, 1965). Second, it makes the stimulus presented on a given trial depend on the responses made on, at most, the two preceding trials, whereas all other rules that estimate points closer to the $75 \%$ point on the psychometric function require a longer block of trials between successive changes in the stimulus level (Wetherill \& Levitt, 1965). The rule selected can be stated as follows: Whenever an incorrect response is made, the stimulus level is raised; however, the stimulus level is not decreased until two consecutive correct responses have been made since the last change in the stimulus level. Thus, once a stimulus value is selected, it is not changed until one of these three patterns of responses occurs: (1) an incorrect response (increase the stimulus level); (2) a correct response followed by an incorrect response (increase the stimulus level); or (3) two consecutive correct responses (decrease the stimulus level).

It is easy to see that the probability of increasing the stimulus level equals the probability of decreasing it at the 0.707 point on the psychometric function. Suppose that a level, i, had just been selected and that the associated point on the psychometric function was $p(i)$. Then, the probability that the next stimulus level will be lower than $i$ is the probability of two correct responses or $\mathrm{p}(\mathrm{i})^{2}$, while the probability that the next stimulus level will be higher than $i$ is the probability of an incorrect response or a correct response followed by an incorrect response, which is $[1-\mathrm{p}(\mathrm{i})]+\mathrm{p}(\mathrm{i})[1-\mathrm{p}(\mathrm{i})]$. The point of stability at which the probability of a stimulus increase is the same as the probability of a stimulus decrease is the point at which

$$
p(i)^{2}=[1+p(i)][1-p(i)] .
$$

The solution to this equation is $p(i)=0.707$.

Two remarks can be made concerning the two rules we have selected. First, the forced-choice rule is more costly than the yes-no rule in the following sense: If a predetermined number of trials, say 50 , is to be run, more changes of stimulus level will be made under the yes-no rule than under the forced-choice rule. This factor should tend to increase the variability of the forced-choice staircase estimates relative to the yes-no staircase estimates when both staircases are run the same number of trials. Second, we could, in principle, use the forced-choice rule in a yes-no situation, in which case we would estimate the 0.707 point on the yes-no psychometric function. However, it makes no sense to use the yes-no rule in a forced-choice situation since there is not just one point, but a continuum of stimulus values for which the forced-choice psychometric function has the value 0.5 (or values essentially equal to 0.5 ).

\section{Step Size and Step Placement}

Before an $E$ begins to run a staircase threshold estimation, he must decide on the location and spacing of the stimuli to be presented. Wetherill (1963) points out that a small step size between adjacent stimuli leads to low variability in staircase estimates with the yes-no procedure, but that under these conditions, bias induced by the intensity of the first stimulus presented is increased. Cornsweet (1962, p. 486) suggests that "the size of the steps should be so selected that no more than two, three, or four like responses are made before a reversal of the response occurs." To follow this prescription, an E should choose a step size so that five or fewer steps completely span the intensity range over which the psychometric function varies. In practice, however, an $\mathrm{E}$ will not know the slope of the psychometric function exactly, and so he must guess at the relationship between the step size he selects and the psychometric function. For this reason, we have run our simulated staircases with several different spacings between adjacent stimuli.

In the case of the linear yes-no psychometric function, the smallest and largest stimuli presented were placed at $I_{0}$ and $I_{1}$, with the associated probabilities of a "yes" response being 0 and 1.0 (i.e., $I_{0}$ and $I_{1}$ are at the ends of the linearly increasing segment of the psychometric function). The remaining stimuli were equally spaced along the interval, $\left(I_{0}, I_{1}\right)$. Several simulations were run, each with a different number of steps between $I_{0}$ and $I_{1}$. The steps were chosen to give equal probability intervals along the psychometric function. The number of intervals between $I_{0}$ and $I_{1}$ took on one of the following values: $2,3,4,5$, or 10 . Thus, for example, in the five-interval case, the six stimuli were located at $I_{0}, I_{1}$ and those intermediate points at which the probability of the yes response was 0.2 , $0.4,0.6$, and 0.8 . No other stimuli were needed because whenever $I_{0}\left(I_{1}\right)$ occurs, the next higher (lower) intensity value must always occur on the following trial.

The problem of locating stimuli along the linear forced-choice psychometric function was handled similarly. Stimuli 
were placed at $I_{0}$ and $I_{1}$ (with response probabilities 0.5 and 1.0 , respectively) and equally spaced between these two points. The number of equal stimulus steps between $I_{0}$ and $I_{1}$ took on one of the following values: $1,2,3,4,5$, or 10 . Because errors are made when intensity $I_{0}$ is presented, stimulus levels below $I_{0}$ are needed; for all such additional stimuli, the probability of a correct response was set at 0.5 .

We next consider the procedure used to determine the location and spacing of stimuli along the cumulative normal psychometric function. Because the staircase rules we have chosen tend to concentrate stimulus presentations approximately in the middle of the interval $\left(I_{0}, I_{1}\right)$, we first selected that normal distribution that has its mean midway between $I_{0}$ and $I_{1}$ and a cumulative distribution function with a slope at the mean that matched the slope of the linear psychome tric function. The resulting ogival psychometric function has a probability of "yes" equal to 0.098 associated with intensity $I_{0}$ and 0.902 associated with $I_{1}$. Just as was done with the linear psychometric function, stimuli were evenly spaced along the intensity continuum between $I_{0}$ and $I_{1}$. For each staircase simulation, additional stimuli were added below $I_{0}$ and above $I_{1}$ using the same spacing between stimuli as was used between $I_{0}$ and $I_{1}$. For both the yes-no and the forced-choice cases, simulations were run with 2,5 , or 10 stimulus intervals between $I_{0}$ and $I_{1}$.

All the methods described above for selecting stimulus intensities have one feature in common: the stimuli are symmetrically located about the midpoint on the interval, $\left(\mathbf{I}_{0}, I_{1}\right)$. Clearly, since an $E$ could not know in advance the exact location of the psychometric function, it would be unlikely that any set of stimuli he selected would be located in this fashion. We conjectured that stimulus location would not significantly influence the bias or variability of staircase estimators. To check this, we ran several asymmetric control simulations with the linear psychometric function. It is clear that stimulus location will have the largest effect on the staircase estimator when the stimulus spacing is large. The largest spacing used in any of the simulations is equal to half the interval, $\left(I_{0}, I_{1}\right)$. For this reason, all these asymmetric control simulations were run with the spacing between adjacent stimuli equal to half the interval from $I_{0}$ to $I_{1}$. This spacing results in one stimulus level below $I_{0}$, two between $I_{0}$ and $I_{1}$, and one above $I_{1}$. For the yes-no case, five control simulations were run with stimuli located so that the associated probabilities of the yes response were: $1.0,0.9,0.4,0.0 ; 1.0,0.8,0.3,0.0$; $1.0,0.75,0.25,0.0 ; 1.0,0.7,0.2,0.0$; or $1.0,0.6,0.1,0.0$. We expect that these simulations will not reveal properties of the estimator significantly different from the simulation for which the stimuli were located at the $1.0,0.5$, and 0.0 points on the psychometric function. In the forced-choice case, four control simulations were run, and the probabilities of a correct response associated with the stimuli selected were: $1.0,0.95,0.70,0.5$, $\ldots ; 1.0,0.90,0.65,0.50, \ldots ; 1.0,0.85$, $0.60,0.50, \ldots$; or $1.0,0.80,0.55,0.50$, Again, we expect that these simulations will be nearly identical in outcome to the forced-choice simulations with stimuli located at $1.0,0.75,0.50$, ...

\section{Initial Stimulus Intensity}

Ideally, one would set the initial stimulus intensity as close as possible to the threshold. In practice, one usually runs a few preliminary trials before starting the staircase. This procedure permits a very rough estimate of the location of the threshold (Cornsweet, 1962). In our simulations, the initial intensity level was chosen from those stimuli in the closed interval, $\left(I_{0}, I_{1}\right)$. Each of these stimuli was selected equally often. Thus, we assumed that the $E$ was likely to start at some stimulus value in the interval over which the psychometric function increases rapidly.

\section{Discarding Initial Trials of the Staircase}

For various reasons, Es often discard a block of trials at the beginning of a staircase run and use the remainder of the data to estimate the threshold (Cornsweet, 1962). In imitation of this practice, we have always excluded the first 10 trials of each staircase run and derived our estimate from the remaining trials.

\section{When to Stop a Staircase Run}

An increase in the number of trials in a staircase run obviously will reduce the variability of the resulting estimator However, trials are costly to the $E$ who may be trying to maintain a constant level of attention and sensitivity in the $S$ and to minimize the time spent making the estimate. Cornsweet (1962) has suggested under some conditions as few as 20 trials can be used to estimate a threshold with a staircase procedure. In order to estimate the bias and variability of the staircase estimator over the range of run lengths typically used by Es, we have simulated runs of length 25,50 , and 200 . Since the first 10 trials of each run are discarded, the number of trials used in deriving the estimate from these runs was, respectively, 15,40 , and 190 trials.

\section{Derivation of Estimate from Data}

One of the features of the staircase methods that appeals to Es is the ease with which the threshold estimate can be derived from the data. Several estimation procedures have been suggested. The simplest of these is to take as the estimate the median over trials of the intensity levels presented. A still simple, but less rapid, method is to determine the mean over trials of the levels presented. Both of these methods are suggested by Cornsweet (1962). Wetherill and Chen (1964) suggest that the intensity levels at which "peaks" and "valleys" occurred be averaged and that this average be taken as the estimate. "Peaks" and "valleys" appear as local maxima and minima on the plot of intensity as a function of trials. More complex statistical treatments of the data have been considered by Dixon and Mood (1948). We have estimated thresholds from our simulated staircase runs by calculating both the median and mean of the intensities presented (excluding the first 10 trials).

\section{METHOD}

Blocks of either 50 or 500 staircase runs were simulated under a given condition. The input to the computer that specified the conditions to be imposed in a given block consisted of the following information: (1) The set of available stimuli and the response probability associated with each. This information specified the psychometric function, the step size, and step placement. (2) The rule to be followed for generating each intensity level from those preceding it. (3) The starting stimuli-i.e., those stimuli in the interval, $\left(\mathrm{I}_{0}, \mathrm{I}_{1}\right)$; the staircase run was always started from one of these intensity levels, and each was selected equally often. (4) The number of trials to be discarded at the beginning of each run. This number was always 10. (5) The number of trials, $N$, to be run in each staircase simulation and the number of repetitions, $M$, of the staircase. The values of $\mathbf{N}$ chosen were 25,50 , or 200 . When 200 trials were run, then 50 staircases were generated; otherwise, $M$ was set equal to 500.

The computer essentially ran itself as a S. On each trial, a "response" was selected by noting the intensity level for that trial and comparing the associated response probability to a random number generated by sampling from a uniform distribution over the interval, $(0,1)$. If the random number was less than the response probability, a "yes" or correct response, as appropriate, was made. Then, on the basis of the stimulus selection rule, the next stimulus was generated and the process repeated until the requisite number, $\mathrm{N}$, of 
Table 1

Simulation of Yes-No Staircases

\begin{tabular}{|c|c|c|c|c|c|}
\hline \multirow{2}{*}{$\begin{array}{l}\text { Number of } \\
\text { Trials Per } \\
\text { Staircase } \\
\end{array}$} & \multirow{2}{*}{$\begin{array}{l}\text { Number of } \\
\text { Intervals } \\
\text { Between } \\
I_{0} \text { and } I_{1}\end{array}$} & \multicolumn{2}{|c|}{ Medians } & \multicolumn{2}{|c|}{ Means } \\
\hline & & $\begin{array}{l}\text { Threshold } \\
\text { Estimate }\end{array}$ & $\begin{array}{c}\text { SD of } \\
\text { Estimatesb }\end{array}$ & $\begin{array}{l}\text { Threshold } \\
\text { Estimatea } \\
\end{array}$ & $\begin{array}{c}\text { SD of } \\
\text { Estimatesb }\end{array}$ \\
\hline 25 & $\begin{array}{r}10 \\
5 \\
4 \\
3 \\
2\end{array}$ & $\begin{array}{l}.495 \\
.499 \\
.493 \\
.492 \\
.498\end{array}$ & $\begin{array}{l}.11 \\
.12 \\
.14 \\
.17 \\
.09\end{array}$ & $\begin{array}{l}.494 \\
.497 \\
.495 \\
.494 \\
.498\end{array}$ & $\begin{array}{l}.10 \\
.11 \\
.11 \\
.10 \\
.09\end{array}$ \\
\hline 50 & $\begin{array}{r}10 \\
5 \\
4 \\
3 \\
2\end{array}$ & $\begin{array}{l}.509 \\
.504 \\
.500 \\
.517 \\
.500\end{array}$ & $\begin{array}{l}.08 \\
.10 \\
.07 \\
.17 \\
.00\end{array}$ & $\begin{array}{l}.503 \\
.499 \\
.496 \\
.502 \\
.503\end{array}$ & $\begin{array}{l}.07 \\
.07 \\
.07 \\
.06 \\
.06\end{array}$ \\
\hline 200 & $\begin{array}{r}10 \\
5 \\
4 \\
3 \\
2\end{array}$ & $\begin{array}{l}.498 \\
.488 \\
.500 \\
.498 \\
.500\end{array}$ & $\begin{array}{l}.05 \\
.10 \\
.00 \\
.17 \\
.00\end{array}$ & $\begin{array}{l}.496 \\
.494 \\
.504 \\
.500 \\
.500\end{array}$ & $\begin{array}{l}.04 \\
.03 \\
.03 \\
.03 \\
.03\end{array}$ \\
\hline
\end{tabular}

"Expressed in terms of corresponding value of the probability of a "yes" response on the psychometric function.

$b$ Expressed as a fraction of the interval $\left(I_{0}, I_{1}\right)$.

Table 2

Simulation of Forced-Choice Staircases

\begin{tabular}{|c|c|c|c|c|c|}
\hline \multirow{2}{*}{$\begin{array}{c}\text { Number of } \\
\text { Trjals Per } \\
\text { Staircase } \\
\end{array}$} & \multirow{2}{*}{$\begin{array}{c}\text { Number of } \\
\text { Intervals } \\
\text { Between } \\
I_{0} \text { and } I_{1}\end{array}$} & \multicolumn{2}{|c|}{ Medians } & \multicolumn{2}{|c|}{ Means } \\
\hline & & $\begin{array}{l}\text { Threshold } \\
\text { Estimatea }\end{array}$ & $\begin{array}{c}\text { SD of } \\
\text { Estimates } b\end{array}$ & $\begin{array}{l}\text { Threshold } \\
\text { Estimate }^{\text {a }}\end{array}$ & $\begin{array}{c}\text { SD of } \\
\text { Estimates b }\end{array}$ \\
\hline 25 & $\begin{array}{r}10 \\
5 \\
4 \\
3 \\
2 \\
1\end{array}$ & $\begin{array}{l}.722 \\
.708 \\
.706 \\
.680 \\
.658 \\
.604\end{array}$ & $\begin{array}{l}.16 \\
.20 \\
.22 \\
.28 \\
.36 \\
.74\end{array}$ & $\begin{array}{l}.722 \\
.708 \\
.703 \\
.678 \\
.660 \\
.564\end{array}$ & $\begin{array}{l}.16 \\
.20 \\
.22 \\
.26 \\
.34 \\
.60\end{array}$ \\
\hline 50 & $\begin{array}{r}10 \\
5 \\
4 \\
3 \\
2 \\
1\end{array}$ & $\begin{array}{l}.712 \\
.700 \\
.692 \\
.678 \\
.675 \\
.500^{c} \mathrm{c}\end{array}$ & $\begin{array}{l}.14 \\
.16 \\
.18 \\
.22 \\
.28 \\
.44\end{array}$ & $\begin{array}{l}.714 \\
.700 \\
.692 \\
.678 \\
.652 \\
.564\end{array}$ & $\begin{array}{l}.12 \\
.16 \\
.16 \\
.20 \\
.26 \\
.38\end{array}$ \\
\hline 200 & $\begin{array}{r}10 \\
5 \\
4 \\
3 \\
2 \\
1 \\
\end{array}$ & $\begin{array}{l}.706 \\
.700 \\
.718 \\
.680 \\
.660 \\
.505 \\
\end{array}$ & $\begin{array}{l}.08 \\
.10 \\
.12 \\
.10 \\
.10 \\
.08\end{array}$ & $\begin{array}{l}.706 \\
.694 \\
.694 \\
.681 \\
.650 \\
.546 \\
\end{array}$ & $\begin{array}{l}.08 \\
.10 \\
.08 \\
.08 \\
.08 \\
.22 \\
\end{array}$ \\
\hline
\end{tabular}

axpressed in terms of corresponding value of the probability of a "yes" response on the psychometric function.

$b$ Expressed as a fraction of the interval $\left(I_{0}, I_{1}\right)$

c Actually 0.1 intensity unit below $I_{1}$.

trials had been run. Finally, the median and the mean intensity level for that run were calculated and stored. This process was repeated until $M$ runs had been completed, at which time the mean and standard deviation of the $M$ individual staircase medians and means were computed and printed out.

\section{RESULTS}

The majority of the simulations were run with the linear psychometric function. Tables 1, 2, and 3 present these results, and Table 4 permits a comparison of the estimator's properties when the responses are generated by an ogival psychometric function to the properties it has when a straightforward evaluation of these predictions, we have presented the average of the $M$ estimates in terms of response probability levels rather than intensity levels. Thus, for example in the last row of Table 1, when the median intensity level was taken as the threshold estimate for each staircase run, the mean of the estimates over the $M$ repetitions of the staircase was the intensity level associated with the 0.500 point on the psychometric function (i.e., halfway between $I_{0}$ and $I_{1}$ ).

The standard deviation of each of the estimators is expressed as a fraction of the interval $\left(I_{0}, I_{1}\right)$. In other words, we have taken the length of the interval, $\left(I_{0}, I_{1}\right)$, as the intensity unit for the purpose of reporting these standard deviations. Tables 1 and 2 present the mean and standard deviation of the threshoid estimates as calculated both from the mean and from the median intensity presented. The data summaries in Tables 3 and 4 are based only on the mean intensity level presented.

In order to evaluate the stability of our estimates, we ran our program through the computer twice for many of the conditions studied. Thus, we replicated these simulations by rerunning the program with different, independent random numbers used to generate the responses. Because the results of each such pair of simulations were very similar, they have been combined. Therefore, except as noted in table footnotes, each row of the four tables for which $\mathbf{N}$ equals 25 or 50 trials is based on 1,000 staircase simulations, and each row with $\mathrm{N}$ equal to 200 trials is based on 100 staircase simulations.

\section{DISCUSSION}

First, we consider what the present analysis reveals concerning four variables: method of deriving the estimate, location

Table 3

Effect of Asymmetric Stimulus Locationsa

\begin{tabular}{|c|c|c|}
\hline $\begin{array}{c}\text { Location of Stimuli } \\
\text { on Psychometric } \\
\text { Function }\end{array}$ & $\begin{array}{l}\text { Threshold } \\
\text { Estimate }\end{array}$ & $\begin{array}{c}\text { SD of } \\
\text { Threshold } \\
\text { Estimates }\end{array}$ \\
\hline \multicolumn{3}{|c|}{ Yes-No Procedure } \\
\hline $1.0,0.5,0.0$ & $.503^{b}$ & $.06^{b}$ \\
\hline $1.0,0.6,0.1,0.0$ & .508 & .07 \\
\hline $1.0,0.7,0.2,0.0$ & .505 & .08 \\
\hline $1.0,0.75,0.25,0.0$ & .500 & .08 \\
\hline $1.0,0.8,0.2,0.0$ & .492 & .08 \\
\hline $1.0,0.9,0.4,0.0$ & .494 & .07 \\
\hline \multicolumn{3}{|c|}{ Forced-Choice Procedure } \\
\hline & .652 & $\begin{aligned} .2 b \\
28\end{aligned}$ \\
\hline $1.0,0.8,0.55,0$ & .647 & .28 \\
\hline $1.0,0.85,0.6,0.5$ & .643 & .28 \\
\hline $1.0,0.9,0.65$, & .643 & .28 \\
\hline $1.0,0.95,0.7,0.5$ & .646 & .28 \\
\hline
\end{tabular}

a All staircases run for 50 trials. Threshold estimates based on mean intensity level on last 40 trials.

$b$ Based on 1,000 replications. All other estimates are based on 500 replications. 
Table 4

Comparison of Ogival and Linear Psychometric Functions ${ }^{a}$

\begin{tabular}{|c|c|c|c|c|}
\hline \multirow{2}{*}{$\begin{array}{c}\text { Number of } \\
\text { Intervals } \\
\text { Between } \\
I_{0} \text { and } I_{1}\end{array}$} & \multicolumn{2}{|c|}{ Ogival Function } & \multicolumn{2}{|c|}{ Linear Function } \\
\hline & $\begin{array}{l}\text { Threshold } \\
\text { Estimate }^{\mathrm{b}}\end{array}$ & $\begin{array}{c}\text { SD of } \\
\text { Estimates }\end{array}$ & $\begin{array}{l}\text { Threshold } \\
\text { Estimate }^{\mathrm{c}}\end{array}$ & $\begin{array}{c}\text { SD of } \\
\text { Estimates }\end{array}$ \\
\hline $\begin{array}{r}10 \\
5 \\
2\end{array}$ & $\begin{array}{l}.497 \\
.501 \\
.504\end{array}$ & $\begin{array}{l}.08 \\
.08 \\
.07\end{array}$ & $\begin{array}{r}\text { Procedure } \\
.503 \\
.499 \\
.503\end{array}$ & $\begin{array}{l}.07 \\
.07 \\
.06\end{array}$ \\
\hline $\begin{array}{r}10 \\
5 \\
2\end{array}$ & $\begin{array}{l}.696 \\
.688 \\
.662\end{array}$ & $\begin{array}{l}.20^{\text {For }} \\
.18 \\
.24\end{array}$ & $\begin{array}{c}\text { Procedure } \\
.714 \\
.700 \\
.652\end{array}$ & $\begin{array}{l}.12 \\
.16 \\
.26\end{array}$ \\
\hline
\end{tabular}

a All staircases run for 50 trials. Threshold estimates based on mean intensity level on last 40 trials.

$b$ Based on 500 replications.

c Based on 1,000 replications.

of stimuli, shape of the psychometric function, and number of trials run.

Tables 1 and 2 permit an evaluation of two methods of deriving a staircase estimate from data-taking the median or the mean of the intensity levels presented on all (but the first 10) trials. It is clear that there is no difference between the average estimates. As might be expected, the variability of the estimates is greater when the median is used rather than the mean. With the yes-no procedure, the variability in the medians is about $50 \%$ higher, on the average, than that in the means; in the forced-choice case, this factor averages $5 \%$. In a few cases in Table 1 , the variability of the medians is less than that of the means. This occurs only when one of the set of stimuli presented lies on the 0.50 point of the psychometric function. Obviously, no experiment could be designed to take advantage of this, and so we conclude that, although more time-consuming, calculation of the mean intensity level results, on the average, in somewhat reduced variability in the staircase estimator.

Table 3 clearly shows that the effect of the location of the stimuli is negligible when the spacing is held constant and equal to half the interval $\left(I_{0}, I_{1}\right)$. Neither the mean nor the variability of the estimator is affected by this factor. The same result can be easily demonstrated analytically for the case of stimulus spacing equal to the entire interval, $\left(I_{0}, I_{1}\right)$. These results agree with our conjecture that this variable does not influence staircase estimates.

Examination of Table 4 reveals that ogival and linear psychometric functions result in estimates with virtually identical means and standard deviations. This is a most fortunate state of affairs, for it gives us confidence that our results are not sensitive to small variations in the shape of the psychometric function.

Finally, we turn to the effect of the number of trials, $\mathrm{N}$, on the variability of the staircase estimator. If successive staircase trials were independent, the effect of increasing the number of trials run by some factor, $\mathrm{k}$, would be to decrease the standard deviation of the estimator by a staircase run are not independent, and so it is not surprising that the reduction in variability is not quite this great. The average reduction that occurred in our simulations can be calculated from the figures in the right-hand columns of Tables 1 and 2 . When the number of trials per run is increased from 25 to 50 , the number of trials on which the estimate is based actually increases from 15 to 40 because the first 10 trials of each run are discarded. In this case, one would expect a $39 \%$ reduction in the standard deviation of the estimate if the trials were independent; however, the average reduction is $35 \%$ for the yes-no condition and $28 \%$ for the forced-choice condition. When the number of trials used to calculate the estimate is increased from 40 to 190 , the reduction expected if the trials were independent is $54 \%$. The reduction found was only $52 \%$ in the yes-no case and $50 \%$ in the forced-choice condition. With the help of these figures, an $\mathrm{E}$ can determine roughly how much he can improve his estimate of a threshold by an increase in the length of the staircase run.

In order to minimize the effects of response biases or patterns of responding, some Es run two or more staircases concurrently (Cornsweet, 1962; Teller, 1965). It is customary to interleave trials from the staircases in an irregular or random order so that the $S$ does not know which staircase any particular trial is drawn from. Such a procedure yields a threshold estimate from each of the interleaved staircases. We are now in a position to answer the following question: If 50 trials are to be run, which procedure leads to a lower variance for the estimator-running a single staircase for 50 trials or running two staircases for 25 trials each? A combined factor equal to $\sqrt{\mathrm{k}}$. Successive trials in a estimate based on two runs of $\mathrm{N}$ trials each will have $29 \%$ smaller standard deviation than a single $\mathbf{N}$-trial estimate. From the comnivits in the preceding paragraph, it is clear that, with the forced-choice procedure, two 25-trial staircases are very nearly as variable as a single 50-trial staircase; however, with the yes-no procedure, two 25-trial staircases are slightly more variable than a single 50 -trial run. Running four 50-trial staircases decreases the standard deviation of the estimate from a single 50-trial run by $50 \%$. Thus, it is clear that the effect of nonindependence is approximately cancelled by the effect of rejecting the first 10 trials, and so there is essentially no difference in variability between a single 200 -trial run and four 50 -trial runs.

The theoretical superiority of forced-choice techniques over yes-no ones for the study of psychophysical thresholds has been discussed in the introduction. Given this superiority, it is unfortunate that our simulations reveal that the statistical properties of the yes-no staircase estimator are, in three respects, better than those for the forced choice. First, the forced-choice estimates are more variable than the yes-no estimates. This can be seen by study of the right-hand columns of Tables 1 and 2. Note that $I_{0}$ is the highest stimulus level below which the $S$ never detects the stimulus, and that $I_{1}$ is the lowest level above which he always detects it. For the purpose of comparing the variability of the two procedures we have assumed that $I_{0}$ and $I_{1}$ are the same whether the yes-no or the forced-choice procedure is used. The standard deviations in Tables 1 and 2 are presented as a fraction of the $\left(I_{0}, I_{1}\right)$ interval. Comparison reveals that the standard deviation of the forced-choice estimates is, on the average, 2.6 times as large as that of the yes-no estimates. 5 The forced-choice technique has many advantages over the yes-no technique; but it is considerably more costly to achieve a specified accuracy in one's estimate of a given point on the psychometric function using the forced-choice technique.

Second, the properties of the forced-choice estimator are a function of the number of stimulus intervals between $I_{0}$ and $I_{1}$, while the yes-no estimates are not. It is clear from Tables 1 and 2 that the yes-no estimator is unbiased, whereas the bias of the forced-choice estimator (away from the .707 level) increases with increasing stimulus spacing. When the interval between adjacent stimuli is $1 / 10$ of the $\left(I_{0}, I_{1}\right)$ interval, the forced-choice estimator is very nearly unbiased, but the bias increases to $30 \%$ of that interval in the 
case of the largest spacing between adjacent stimuli. The bias is consistently negative (i.e., the estimates are below the anticipated estimate). In addition, there is a second effect of stimulus spacing that appears only in the forced-choice case; that is, the variance of the estimator is an increasing function of stimulus spacing. This effect can be seen by examination of the right-hand columns of Tables 1 and 2 . These results indicate an effect on the forced-choice estimates of a variable over which an $\mathrm{E}$ may have little control before beginning to collect staircase data.

There is a third respect in which the yes-no staircase procedure is preferred to the forced choice. With the yes-no procedure, it is sometimes possible to identify a staircase nun that is very likely to lead to a biased estimate, but it is much less likely that such an identification can be made if the forced-choice procedure is used. Consider the following hypothetical experiment in which such a biased estimate would arise. Suppose that an E had chosen very large spacing between adjacent stimuli, and that one stimulus was set at $I_{0}$ and the next higher stimulus was set at twice the intensity of $I_{1}$. Then, with the yes-no procedure, the $S$ would alternate "yes" and "no" responses, and the staircase estimate would be $I_{1}$ an estimate with a positive bias due to the stimuli that happened to be selected. Thus, when staircase data consist only of trials at a very few (two or three) stimulus, levels, the E should consider rerunning the staircase with smaller intervals between adjacent stimuli, for it is quite likely that the stimulus spacing is so large that a biased estimate will result. Now consider a forced-choice staircase run with the same choice of stimuli. Once again, the estimate would be biased high, but the $S$ would be presented with a larger variety of stimuli, most of which would be below $\mathrm{I}_{0}$. Thus, this staircase run would appear less stable, and so it would be more difficult to detect the problem of overly large stimulus intervals than it was when the yes-no procedure was used.

We conclude with a reminder to the reader that our conclusions are based on study of a simple (and probably overly simple) stat-S. In particular, we have assumed the existence of no sequential dependencies between responses and of stability in time of the response probabilities (i.e., no learning, fatigue or attention shifts). In addition, we assumed that our stat-S was capable of holding his bias constant, that his proportion of "yes" responses tended to zero with reduction in stimulus intensity, and that the length of the interval $\left(I_{0}, I_{1}\right)$ is the same with either the yes-no or the forced-choice procedure. Violations of each of these assumptions may be found to some degree one place or another in the psychophysical literature. Insofar as real Ss differ from our stat-S, caution should be used when our results are applied.

\section{REFERENCES}

BLOUGH, D. S. Method for tracing dark adaptation in the pigeon. Science, 1955, 121, 703-704.

BROWN, A. E. Measurement of auditory thresholds. Journal of the Acoustical Society of America, 1965, 39, 86-92.

CAMPBELL, R. A., \& LASKY, E. Z. Adaptive threshold procedures: BUDTIF. Journal of the Acoustical Society of America, 1968, 44, 537-541.

CORNSWEET, T. N. The staircase method in psychophysics. American Journal of Psychology, 1962, 75, 485-491.

CORNSWEET, T. N., \& PINSKER, H. M. Luminance discrimination of brief flashes under various conditions of adaptation. Journal of Physiology, 1965, 176, 294-310.

DIXON, W. J., \& MOOD, A. M. A method for obtaining and analyzing sensitivity data. Journal of the American Statistical Association, 1948, 43, 109-126.

FEENY, S., KAISER, P. K., \& THOMAS, J. P. An analysis of data gathered by the staircase method. American Joumal of Psychology, $1966,79,652-654$.

GREEN, D. M., \& SWETS, J. A. Signal detection theory and psychophysics. New York: Wiley, 1966.

HEINEMANN, E. G. The relation of apparent brightness to the threshold for difference in luminance. Journal of Experimental Psychology, 1961, 61, 389-399.

LEVITT, $H$. Discrimination of sounds in hearing. Unpublished doctoral dissertation, University of London, 1964.

POLLACK, I. Methodological determination of the PEST (Parameter Estimation by Sequential Testing) procedure. Perception \& Psychophysics, 1968, 3, 285-289.

SEMB, G., \& SASLOW, M. G. Conformity to experimenter-determined, pay-off enforced, criterion levels in the method of random staircases. Psychophysics Laboratory Report PRP-21A, University of Washington, 1966.

STEVENS, S. S., MORGAN, C. T., \& VOLKMANN, J. Theory of the neural quantum in the discrimination of loudness and pitch. American Psychologist, 1941, 54, 315-335.

STUCKEY, C. W., HUTTON, C. L., \&
CAMPBELL, R. A. Decision rules in threshold determination. Journal of the Acoustical Society of America, 1966, 40, 1174-1179.

TAYLOR, M. M., \& CREELMAN, C. D. PEST: Efficient estimates on probability functions. Journal of the Acoustical Society of America, $1967,41,782-787$.

TELLER, D. Y. The influence of borders on increment thresholds. Unpublished doctoral dissertation, University of California at Berkeley, 1965 (available from University Microfilms, Order No. 66-3710).

THOMAS, J., PADILlA, G., \& ROURKE, D. Spatial interactions in identification and detection of compound visual stimuli. Vision Research, 1969, 9, 283-292.

WETHERILL, G. B. Sequential estimation of quantal response curves. Journal of the Royal Statistical Society, Series B, 1963, 25, 1-48.

WETHERILL, G. B., \& CHEN, H. Sequential estimation of quantal response curves. II. A new method of estimation. Bell Telephone Laboratories Technical Memorandum, 1964.

WETHERILL, G. B., \& LEVITT, H. Sequential estimation of points on a psychometric function. British Journal of Mathematical \& Statistical Psychology, 1965, 18, 1-10.

\section{NOTES}

1. The research was supported by Contract N00014-67-A-0108-0005 between the Office of Naval Research, Physiological Psychology Program, and Columbia University, PHS Grant EY 08070-01 from the National Eye Institute, and by PHS Grant MH 17761-01 from the National Institute of Mental Health. Computer funds were provided by the Department of Psvchologv. University of Washington.

2. Address: Department of Psychology, University of Washington, Seattle, Washington 98105.

3. We wish to thank James P. Egan, Eric Holman, and James P. Thomas for valuable comments on the final manuscript and Margaret Liston for her help with programming.

4. This paper is phrased in terms of threshold measurement. Hov'ever, staircase methods, and therefore our results, have a variety of other applications, both within and outside of the field of psychophysics. For example, variants of these methods could be used to measure the point of subjective equality in a brightness experiment or to estimate lethal dosages of drugs.

5 . We have not found a way of predicting that the foreed-choice cstimates will be so much more variable than those found with the yes-no procedure. We have, however, identified two factors that contribute to the difference. First, there are about $70 \%$ more changes of level made for a given number of trials with the yes-no procedure than with the forced-choice method. Second, intensity levels below $l_{0}$ can be presented when the forced-choice method is used, but this does not occur in the yes-no case.

(Accepted for publication January 21, 1970.) 